\title{
RESEARCH ON PUBLISHING SYSTEM OF FRUIT TREE DISEASES AND INSECT PESTS BASED ON WEBGIS
}

\author{
Zhifang Yang ${ }^{1}$, Gang Liu ${ }^{2}$, Yongsheng $\mathrm{Si}^{1}$ \\ 1 Hebei Agricultural University, 071001, Baoding, China \\ 2 China Agricultural University, 100083, Beijing, China
}

\begin{abstract}
A web publishing system of fruit tree diseases and insect pests was developed based on WebGIS. It can publish most of the new information of fruit tree diseases and insect pests with the spatial and zone features in the orchard. It can also make appropriate prevention and cure decision in time. This system provides many analysis methods, including spatial query, statistical analysis, buffer analysis, overlay analysis, predication analysis, expert diagnosis and so on. The system is developed as the B/S structure with Java Based on GIS platform ArcIMS.
\end{abstract}

Key words: publishing system, WebGIS, buffer analysis, ArcIMS

\section{INTRODUCTION}

The fruit tree diseases and insect pests in our country have occurred frequently in the past decade. The pesticide is the main means to control fruit tree diseases and insect pests, but it influences the fruit quantity badly and endangers the human health. Every country in the world has begun to pay more and more attention to it. In recent years, some expert systems for fruit tree diseases and insect pests were completed in our country. Because the occurrence and development of fruit tree diseases and insect pests have strong regionality, the expert systems did not work very well. Therefore, the technology based on ' $3 \mathrm{~S}$ ' has become the principal research direction in the management about fruit tree diseases and insect pests in our country. 
Fruit tree diseases and insect pests firstly occur in some partial areas and then spread quickly and unevenly to neighborhood by the affect of weather conditions. These statuses of fruit tree diseases and insect pests are related to geography spatial position and are easily expressed by spatial analysis of GIS. The buffer analysis of GIS can help to analyze the spreading tendency and spatial distribution for fruit tree diseases and insect pests in a period. The overlay analysis model can help to analyze the spatial and temporal developing tendency for fruit tree diseases and insect pests in a district and find the main weather factors that are related to fruit tree diseases and insect pests.

The GIS platform ArcIMS was used to develop a publishing system of fruit tree diseases and insect pests based on the B/S structure with Java. The system can publish the most new information for fruit tree diseases and insect pests and can make appropriate prevention and cure decision in time. The system was demonstrated in Yantai city, Shandong Province.

\section{SYSTEM DESIGN}

\subsection{System Framework}

In recent years, the Internet has emerged as an extremely popular and effective means of sharing information, data, and technology. The tremendous growth in Internet use has resulted in an increased demand for the delivery of geographic data, maps, and applications over the Internet. A system is built on Windows 2000 operating system based on WebGIS technology to meet this growing demand. It is designed with a typical three-tier $\mathrm{B} / \mathrm{S}$ (Browse/Server) and MVC (Model-View-Controller) design pattern and is particularly suitable for handling complicated user-interactive circumstance.

The typical three-tier $\mathrm{B} / \mathrm{S}$ system structure (Fig.1) is composed of data layer, logic layer, and view layer, among which database oracle9i is used

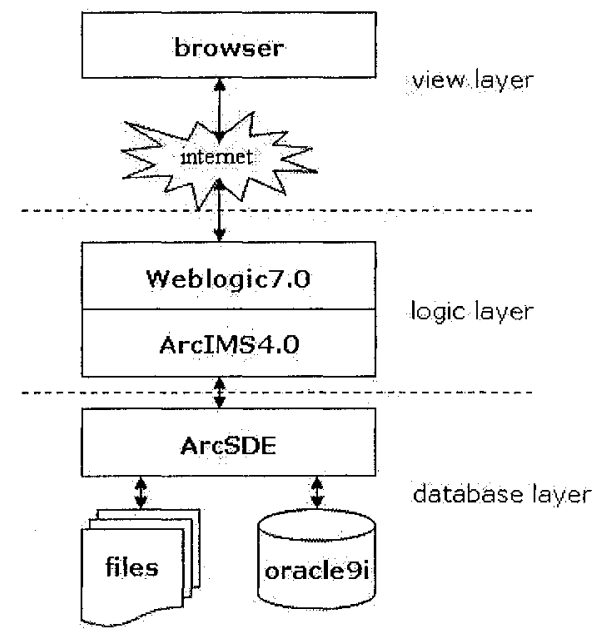

Fig. 1 Three-tier system structure 
for storing attribute data and spatial data and ArcSDE belongs to data layer. Logic layer includes ArcIMS server with Java technology and Weblogic used for Web server. ArcIMS is Internet Map Server software for building and delivering maps, data, designing, publishing, and administering mapping applications over Internet. It takes advantage of the Internet technology that makes it possible to share information and data with many users, either locally or around the world. With ArcIMS, large amounts of area map, data, and application service about fruit tree distribution of Yantai city can be built. ArcSDE works with oracle9i, it is the tool that allows to store and to manage spatial data related to fruit tree diseases and insect pests in orchard in the DBMS. It plays a fundamental role in a multi-user GIS, with which, ArcIMS can work directly with spatial data managed in DBMS.

Java MVC (Fig.2) design pattern allows the Web page to be mostly separated from the internal application code (Controller/Model) and makes it easier to focus on respective function. The Controller is responsible for allocating HTTP requests to the appropriate Model based on configuration mappings. The Model contains the business logic for the application. The Controller forwards the request to the appropriate View component, which is usually implemented by a combination of HTML with JavaScript and CSS. Java MVC design pattern is used to set up the system framework for data

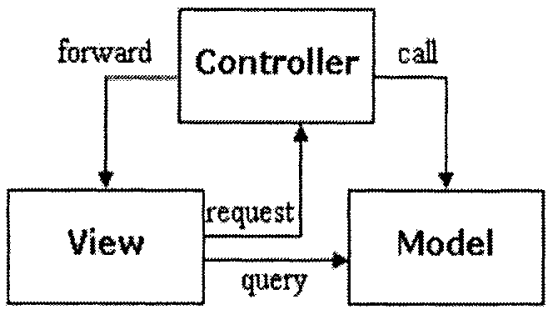

Fig. 2 MVC design mode collecting, managing, analyzing and viewing.

\subsection{System Main Function Modules}

According to actual need, the system includes six big function models, shown as Fig.3:

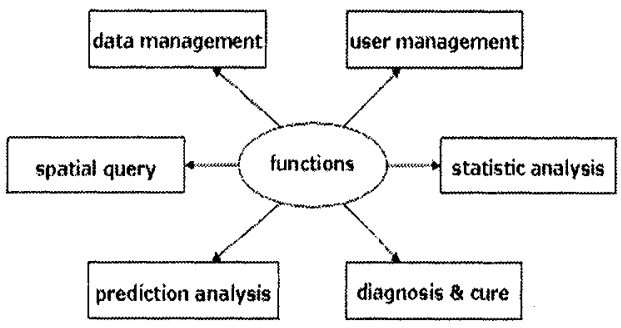

Fig.3 System functions 
1. Data Management: the system needs the supports of multi-source data, which can be accessed from geographical spatial database, meteorological database, knowledge database of fruit tree diseases and insect pests and its real-time monitoring data.

2. User management: the users are classified as common users and administrators. The common users only have the browse right such as query, analysis, etc. But administrators not only have above right but also have right to manage data, including update, import, delete, and so on.

3. Spatial query: In this function model, map query is provided. A given zone is selected by clicking or drawing rectangle in map to get information for diseases and insect pests in orchard related to the selected zone. Besides, the attribute condition query is also included in this model. The information can be accessed after setting special conditions. The last query is the knowledge of fruit tree diseases and insect pests in orchard.

4. Statistics analysis: The aim of statistic analysis is mainly to sum up the statistics, which can create a geographical distribution map of different levels of infection, a statistic map of different infection categories and a bargraph map of the development trend of infection. Figure 4 shows the statistics analysis results.
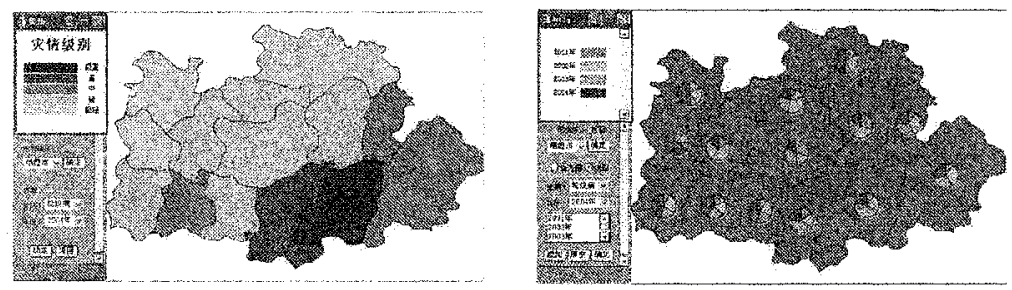

Fig 4: Statistics analysis (classification analysis and bar diagram statistic)

5. Spatial analysis: The spatial analysis for fruit tree diseases and insect pests was completed by Arc/Info. The buffer analysis model can create a zone area by the analysis of the entities of dot, line and polygon. The zone area can indicate the affect range or grade of these entities to their neighbors. Fruit tree diseases and insect pests firstly occur in some partial areas, then spread quickly and unevenly to neighborhood with the affect of weather conditions such as temperature, humidity, wind speed and wind direction etc. 
The buffer analysis model can help to analyze the spreading tendency and spatial distribution for fruit tree diseases and insect pests in a period.

In buffer model, the basic idea is to give a spatial area, then ascertain its adjacent area which size is relative to radius B (Fig 5). So definition of buffer size is showed as follows:

$$
B_{i}=\left\{x: D\left(x, O_{i} \leq d\right)\right\}
$$

where $O=\left\{O_{i}: i=1,2, \cdots, n\right\}$

The spatial buffer area is aggregate values, as shown below:

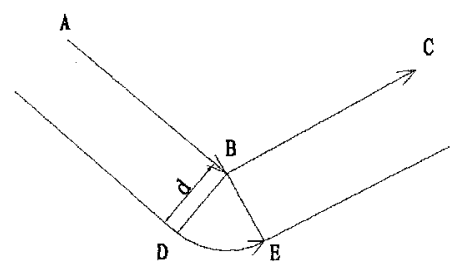

Fig 5: Salient buffer

$$
B=\bigcup_{i=1}^{n} B_{i}
$$

5. Prediction analysis: Some conditions such as weather data are the decisive factors that affect the occurrence of fruit tree diseases and insect pests. The real-time monitored weather data and fruit tree diseases and insect pests data are processed with forecasting models and GIS overlay analysis to give the prediction results. To ensure the precision of forecasting and decision-making, data should be real-time and the high-precision prediction models and decision-making models should be built.

6. Diagnosis and cure: The diagnosis of fruit tree diseases and insect pests is achieved remotely by using the knowledge database and diagnosis model by Internet. The position of fruit tree was firstly selected, the corresponding pictures and text was queried, the occurrence grade of the disease in a town was finally ascertained. Figure 6 shows the map of the occurrence grade of a disease of fruit tree. 


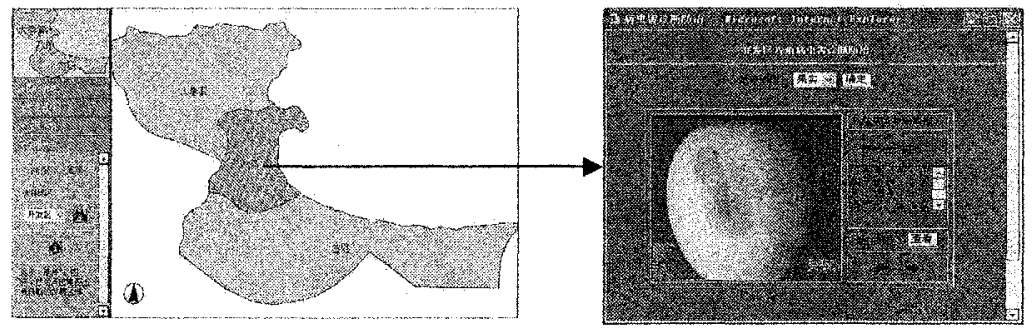

Fig 6: Diagnosis and cure

\section{CONCLUSION}

The system based on WebGIS are developed using GIS software (ArcIMS, ArcSDE, ArcInfo), Web server (Weblogic) and database management system (oracle9i). The system also integrates Java MVC design pattern technology, three-tier $\mathrm{B} / \mathrm{S}$ structures, spatial analysis models, experts knowledge models and the information of fruit tree diseases and insect pests in orchard. It can solve some problem about fruit tree diseases and insect pests in orchard by applying incorporated WebGIS technology. The GIS and biologic geographical statistics are applied to describe the distribution pattern of fruit tree diseases and insect pests and to carry out spatial overlay analysis and so on.

The WebGIS technology is a promising mode to develop a control system for fruit tree diseases and insect pests, newer ArcIMS technology with more actual functions and analysis methods have been developed. It is necessary that some exact decision-making and prediction models should be researched and applied to ensure usability of the system.

\section{ACKNOWLEDGEMENTS}

The research project is founded by the 863 Program- the long-distance diagnosis platform of plant diseases and insect pests based on network 2002AA243031. 


\section{REFERENCES}

[1]Wang Maohua. Possible adoption of precision agriculture for developing counties at the threshold of the new millennium. [J]. Computers and Electronics in Agriculture. 2001, 30:45-50.

$[2]\{0><\} 0\{>$ Wu Lun, Liu Yu, Ma Xiujun, Wei Zhongya, and Tian Yuan. 2001. The Geographic Information System- Its Principle, Methods and Application. China,Beijing:The Science Press. $<0\}$.

[3] $\{0><\} 0\{>$ Mao Feng, Sheng Xiaohua, Ai Lishuang.2001.Development and Pracetice of ArcGIS 8, 193-217.China,Beijing: The Science Press.

[4]Liu Shuhua, Yang Xiaohong, Jiang Wenke and Cao Keqiang. 2003.GIS-based Decision Support System for Crop Pest Control. The Transaction of Agricultural Engineering. 19(4): $147-150$.

[5]Hardy Pundt, Klaus Brinkkotter-Runde. Visualization of spatial data for field based GIS. [J]. Computer \& Geosciences. 2000, 26:51-56

[6] M.A Kramers, C.G.M. Conjin, C. Bastiaansen. An expert system for diagnosing flowerbulb diseases, pests and non-parasitic disorders. Agricultural systems, Vol58, No.1, (1998)PP 57-85.

[7] Lo.Tedeschi, D.G.Fox, P.J.Guiroy: A Decision Support System to Improve Individual Cattle Management. 1. A Mechanistic, Dynamic Model for Animal Growth. Agricultural System. Vol.79(2)(2004)171-204.

[8] M.P.Bange, S.A.Deutscher, D.Larsen, D.Linsley, S.Whiteside: A Handheld Decision Support System to Facilitate Improved Insect Pest Management in Australian Cotton Systems. Computers and Electronics in Agriculture. Vol.43(2)(2004)131-148 